\section{Self-harm and depressive disorder}

SIR: Ennis et al (Journal, January 1989, 154, 41-47)

found a $31 \%$ point prevalence of major depressive episode (MDE) in their sample using DSM-III criteria. We conducted a similar study at Reims Hospital's Emergency Unit with 113 deliberate selfharm patients consulting a psychiatrist (Théret et al, 1988). A $58 \%$ point prevalence of MDE was found using the Structured Clinical Interview for DSM-III-R (SCID).

As discrepant results may arise from different methods, we would like to discuss some points. The DSM-III-R criteria for MDE are known to be more stringent than those of DSM-III, so the discrepancy of results cannot be explained by the use of different classifications. In our study the cases of self-harm were restricted to drug self-poisonings, but it is known that the latter account for approximately $90 \%$ of the former. The sample of Ennis et al's study might not be representative of the patients consulting in psychiatry after a deliberate self-harm at Toronto Hospital, since only $23 \%$ (71 of 251 ) of the patients admitted for that cause at the Emergency Department entered the study. The inter-rater diagnostic reliability was low $(\chi=0.445)$ despite the use of a checklist containing the DSM-III criteria for MDE. A standardised diagnostic assessment tool would have been more appropriate. The results are thus of less practical value. Finally, an astonishing statement by the authors is that attempted suicide and parasuicide are synonymous terms. Such an assimilation could lead to a confusion in the definitions. Kreitman's definition of parasuicide (Dyer \& Kreitman, 1984) does not take suicidal intent into account, so that parasuicidal (or deliberate non-fatal self-harm) people constitute a much broader group than suicide attempters.

LAURENT THÉRET

Clinique Universitaire de Psychiatrie

J. G. Pascalis

C.H.U. - Hôpital Robert Debré

Rue Alexis Carrel

51092 Reims Cedex, France

\section{References}

DYER, J. A. T. \& KrettMaN, N. (1984) Hopelessness, depression and suicidal intent in parasuicide. British Journal of Psychiatry, 144, 127-133.

Theret, L., Pire, J. C. \& PAsCalis, J. G. (1988) Consumption of psychotropic drugs and medical care among 63 depressed patients-major depressive episode following the DSMIII-R criteria - before a drug self-poisoning. Annales MedicoPsychologiques (in press).

Brain white-matter lesions and psychosis

SIR: The phenomenon of late-adult onset psychosis has long posed one of the more vexing problems in neuropsychiatric practice and research. Past investigative efforts have focused on the relationship of this aetiologically heterogeneous syndrome to cognitive disability, structural brain disease, and perceptual, in particular auditory, impairment. Miller et al (1989) have made a substantial advance in our understanding of late-life psychosis via their recent discovery of extensive sub-frontal white matter lesions by magnetic resonance imaging in almost $20 \%$ of the sample studied. The precise mechanism by which the demonstrated subcortical frontal damage might induce a psychotic process remains open to speculation. Like Miller et al, we have been struck by the coincidence of white matter disease and psychotic symptomatology. Other examples include metachromatic leukodystrophy, vitamin $B_{12}$ deficiency, and certain cases of multiple sclerosis. We are writing to draw attention to a hypothesis we recently put forward in an attempt to explain the induction of psychiatric disturbance by white matter damage (Merriam et al, 1989). In brief, we have suggested that deep frontal white-matter lesions may compromise the integrity of certain corticofugal fibres originating in prefrontal cortex which serve to inhibit the mesolimbic dopaminergic system. Disinhibition of this system is in turn speculated to manifest as a psychotic process. There is substantial evidence that damage to the prefrontal cortical cell bodies from which these fibres originate causes pathological excitation of mesolimbic transmission (Pycock et al, 1980). Weinberger (1987) has speculated that idiopathic schizophrenia is the result of a similar process which, although congenital, does not manifest until the brain's dopaminergic systems mature and come 'on line'. We suggest that frontal white matter lesions may induce an acquired version of the same process and thereby surface as a psychotic disturbance.

Our hypothesis posits a causal cascade whereby acquired regional brain dysfunction induces a derangement of neurotransmitter systems traversing those regions; this neurochemical alteration in turn manifests itself as a psychosyndrome which is a phenocopy of an idiopathic psychiatric disorder. The hypothesis put forward to explain the onset of major depression after cerebral infarction posits a similar sequential relationship. Robinson et al (1984) have demonstrated that the incidence of post-stroke depressive episodes bears a direct relationship to damage to a particular brain region, in this case the left frontal lobe. Such lesions are thought to compromise ascending adrenergic pathways and thereby induce a depressive syndrome. We submit that the identification of these sorts of sequential causal relationships, whereby central nervous system lesions evoke neurochemical alterations which in 